\title{
Dimorphisme sexuel et caractérisation biométrique de la Sériole guinéenne, Seriola carpenteri, (Mather 1971) échantillonné dans la Zone Economique Exclusive (ZEE) de la Côte d'Ivoire
}

\author{
Laizih Yves-Armand ATTEMENE ${ }^{1 *}$, Yao Laurent ALLA $^{2}$, Jean Noel YAPI ${ }^{1}$, \\ Melecony Célestin BLE$^{2}$ et Kouakou $\mathrm{YAO}^{1}$ \\ ${ }^{1}$ Laboratoire de Biologie et Cytologie Animales, UFR-SN, Université Nangui Abrogoua (Ex-Université d'Abobo- \\ Adjamé), 02 BP 801 Abidjan 02, Côte d'Ivoire. \\ ${ }^{2}$ Centre de Recherches Océanologiques, Département Aquaculture, BP V 18 Abidjan, Côte d'Ivoire. \\ *Auteur correspondant ; E-mail : yattemene@gmail.com
}

\begin{tabular}{lll}
\hline Received: 24-01-2021 & Accepted: 25-04-2021 & Published: 30-04-2021 \\
\hline
\end{tabular}

\section{RESUME}

La reproduction artificielle des poissons en captivité nécessite la connaissance de leur dimorphisme sexuel. La détermination du dimorphisme sexuel de la Sériole Guinéenne, Seriola carpenteri est difficile à travers l'examen de l'orifice urogénital. Cette étude a pour but de déterminer le dimorphisme sexuel et les caractéristiques biométriques chez Seriola carpenteri (Mather, 1971) échantillonnée dans la Zone Économique Exclusive (ZEE) ivoirienne, dans le Golfe de guinée. Ainsi, un échantillonnage de 360 spécimens de Seriola carpenteri (Mather, 1971) encore appelé Sériole guinéenne a été effectué en raison de 30 individus par mois pendant la période allant d'août 2017 à juillet 2018. Le poids et la longueur standard des spécimens variaient respectivement de 300,2 g à $935 \mathrm{~g}$ et de $22,8 \mathrm{~cm}$ à $36,2 \mathrm{~cm}$. L'étude biométrique basée sur les caractères morphométriques et méristiques n'a enregistré de difference significative et ont révélé une allométrie minorante de la relation taille-poids et une isométrie de relation pour l'essentiel des paramètres linéaires par rapport à la longueur standard (LS). Par ailleurs, les relations morphométriques chez la sériole guinéenne ont montré que la vitesse de croissance de la longueur standard est plus rapide que la vitesse de la croissance des paramètres linéaires. Cette étude a montré qu'il n'existe aucun dimorphisme sexuel chez la sériole guinéenne dans la ZEE ivoirienne.

(C) 2021 International Formulae Group. All rights reserved.

Mots clés : Dimorphisme sexuel, Biométrie, Seriola carpenteri, ZEE, Côte d'Ivoire.

\section{Sexual dimorphism and Biometric characterization of the Guinean amberjack, Seriola carpenter, (Mather 1971) sampled in the Exclusive Economic Zone (EEZ) of Côte d'Ivoire}

\begin{abstract}
Artificial reproduction of fish in captivity requires knowledge of their sexual dimorphism. Determining the sexual dimorphism of the Guinean amberjack, Seriola carpenteri is difficult by examining the urogenital orifice. The aim of this study is to determine the sexual dimorphism and biometric characteristics in Seriola
\end{abstract}


carpenteri (Mather, 1971) sampled in the Ivorian Exclusive Economic Zone (EEZ), in the Gulf of Guinea. Thus, a sampling of 360 specimens of Seriola carpenteri (Mather, 1971) also called Guinean amberjack was carried out at 30 individuals per month during the period from August 2017 to July 2018. The standard weight and length of the specimens varied respectively from $300.2 \mathrm{~g}$ to $935 \mathrm{~g}$ and from $22.8 \mathrm{~cm}$ to $36.2 \mathrm{~cm}$. The biometric study based on morphometric and meristic characters did not record any significant difference and revealed a lower allometry of the height-weight relation and an isometry of relation for the main part of the linear parameters compared to the standard length (LS). Moreover, morphometric relationships in Guinean amberjack have shown that the growth rate of the standard length is faster than the growth rate of the linear parameters. This study showed that there is no sexual dimorphism in Guinean amberjack in the Ivorian EEZ.

(C) 2021 International Formulae Group. All rights reserved.

Keywords : Sexual Dimorphism, Biometric, Seriola carpenteri, EEZ, Côte d'Ivoire.

\section{INTRODUCTION}

En Côte d'ivoire, le secteur des pêches et de l'aquaculture occupe une place stratégique dans l'économie au regard de la problématique de la sécurité alimentaire. Ce secteur représente $3,1 \%$ du PIB agricole et 0,74\% du PIB total (FIRCA, 2013). Selon la même source, la consommation nationale de poisson est estimée à 320000 tonnes/an pour une production locale moyenne de 42102 tonnes en 2010. En Atlantique Est, les espèces les plus exploitées aussi bien par la pêcherie industrielle qu'artisanale sont les thonidés. Ces espèces sont simultanément capturées en association avec d'autres espèces de poissons désignées sous le terme « capture accessoire » c'est à dire la capture d'espèces non ciblées, parmi lesquelles se retrouve la sériole Guinéenne (Seriola carpenteri). Seriola carpenteri (Mather, 1971) est un poisson qui appartient à la famille des Carangidés. Elle se rencontre principalement le long des côtes ouest-africaines, de l'Angola au Cap-Vert, à Dakar, ainsi que dans la Méditerranée (Golani, 2002). Cette espèce est de plus en plus présente dans les captures de la pêcherie et dans les habitudes alimentaires des populations de beaucoup de pays. En effet, dans les aires marines telles que l'Iles de Lampedusa dans la Méditerrané la reproduction de l'espèce a été étudiée (Pizzicori et al., 2000). Aussi, un certain nombre d'auteurs se sont penchés sur les caractères morphologiques de la sériole Guinéenne respectivement dans l'Atlantique Nord-Est, la Méditerrané et le Nord-Est de la Tunisie (Quéro, 1986 ; Louisy, 2015 ; Capapé et al., 2018). En aquaculture, l'approvisionnement en semences doit être assuré pour le développement économique de ce secteur (Ouedraogo et al., 2015). La propagation naturelle seule de certains poissons ne peut assurer un élevage en captivité (Mylonas et al., 2010). La reproduction est essentielle à la conservation des espèces (Niass et Fall, 2015) et le contrôle de la reproduction artificielle des poissons est essentiel pour promouvoir l'aquaculture (Kamelan et al., 2013). La propagation artificielle du poisson nécessite un contrôle de certains paramètres inclus le dimorphisme sexuel (Bijaksana et al., 2015). Certains poissons de la famille des Carangidae sont difficiles à distinguer sexuellement dont la sériole Guinéenne. L'étude biométrique est basée sur le traitement morphologique et méristiques des données variables mesurées sur des échantillons entre autres les poissons. Les caractères morphologiques et méristiques sont importants dans la mesure où ils permettent d'identifier les espèces de poissons et leurs particularités d'habitats ainsi que les critères applicables à tout cours d'eaux, lacs ou mers (Karatas, 2005). En Côte d'Ivoire, les données sur la morphologie de Seriola carpenteri pêchée dans la Zone Economique Exclusive (ZEE) sont quasi inexistantes. Pourtant, la part de cette espèce dans les débarquements de la pêche artisanale est non négligeable bien qu'elle ne soit pas la cible première des acteurs de cette pêche. Par ailleurs, l'intérêt grandissant que les populations accordent à ce poisson suscites des interrogations quant à la survie et l'état d'embonpoint de cette espèce. Le but de la présente étude était de déterminer les 
caractéristiques morphométriques de la sériole guinéenne échantillonnée dans la ZEE afin de mettre à la disposition de la communauté scientifique une base de données pour une éventuelle gestion du stock.

\section{MATERIEL ET METHODES \\ Zone d'étude}

Cette étude a été réalisée au niveau de la Zone Economique exclusive de la Côte d'Ivoire, dans le golfe de Guinée (Océan Atlantique). La ZEE ivoirienne s'étend du cap des Palmes $\left(8^{\circ} \mathrm{W}\right)$ à l'ouest jusqu'au cap des Trois-Pointes $\left(2^{\circ} 30^{\prime} \mathrm{W}\right)$ à l'est (Diaha et al., 2010), sur une longueur d'environ 600 kilomètres (Figure 1). Elle est caractérisée par une forte variabilité des paramètres climatologiques (vent, pluviométrie) et océanographiques (température, salinité, niveau moyen, oxygène dissous, courants) (N'Goran et al., 2001).

\section{Echantillonnage}

Un échantillon de 360 spécimens de Seriola carpenteri a été effectué en raison de 30 individus par mois sur une période de 12 mois allant d'août 2017 à juillet 2018 . L'ensemble des 360 individus étaient issus des captures de la pêche maritime artisanale au niveau de la Zone Economique Exclusive (ZEE). Ainsi chaque mois, les spécimens de Seriola carpenteri échantillonnés, étaient convoyés au laboratoire d'Anatomie pathologie (AnaPath) du Centre Hospitalier Universitaire de Treichville pour une étude histologique de la gonade afin d'identifier les mâles des femelles. Cependant, avant toute dissection, les individus ont été numérotés de 1 à 30 et identifiés en fonction du mois de collecte. Ensuite, chaque individu a été soumis à des mesures pondéraux à l'aide d'une balance électronique de marque SARTORUS ${ }^{\mathrm{TM}}$ MSE623S-000-DE, de portée $500 \mathrm{~g}$ et de précision $0,01 \mathrm{~g}$. Aussi, le poids total (PT), les paramètres linéaires telles que la longueur totale (LT), la longueur standard (LS), la longueur à la fourche (LF), la longueur de la tête (LTêt), la longueur du museau (Lm), la largeur du corps (Lar), le diamètre de l'œil (Do) et la longueur des différentes nageoires notamment, la petite et la grande nageoire dorsale, la nageoire pectorale, la nageoire pelvienne, la nageoire anale et la nageoire caudale) ont été mesurés à l'aide d'un ichtyomètre gradué au $\mathrm{mm}$ près. La Figure 2 présente les différents paramètres mesurés. Une fois l'étude histologique terminée et à partir de l'observation des coupes histologiques, les spécimens ont été regroupés par sexe. Ensuite, les données issues des différentes mesures ont été soumises à la loi d'allométrie simple de Le Cren (1951) pour la détermination de l'allométrie de croissance à travers équation: $y=a x^{\mathrm{b}}$. Par une transformation logarithmique, cette équation est ramenée à la forme $: \log \mathrm{y}=\log \mathrm{a}+\mathrm{b} \log$ $x$. Dans cette équation (b) est le coefficient d'allométrie encore appelé pente ou coefficient de croissance. La comparaison à l'aide du test $\mathrm{t}$ de Student à $5 \%$ comme seuil d'erreur de la valeur observée de (b) à la valeur théorique 1 ou 3 selon qu'il s'agisse de relation allométrique liant deux paramètres linéaires ou de relation allométrique reliant un paramètre linéaire à un paramètre pondéral. Ainsi, dans le cas de relation liant deux (2) paramètres linéaires, quand $b=1$ la croissance est dite isométrique; quand $\mathrm{b}<1$ la croissance est minorante et quand $\mathrm{b}>1$ la croissance est majorante. Cependant, lorsque la relation lie un paramètre linéaire à un paramètre pondéral, la croissance est dite : isométrique quand $\mathrm{b}=3$; minorante quand $\mathrm{b}<3$ et majorante quand $\mathrm{b}>3$.

\section{Analyses statistiques}

Le logiciel utilisé pour les analyses statistiques est le logiciel STATISTICA version 7.1. Ainsi, les différents coefficients de croissance calculés ont été comparés aux valeurs théoriques de la pente $\mathrm{b}$ à l'aide du test $t$ de Student afin d'apprécier si la différence est significative entre ces deux coefficients. Les différences sont considérées significatives à $\mathrm{p}<0,05$. 


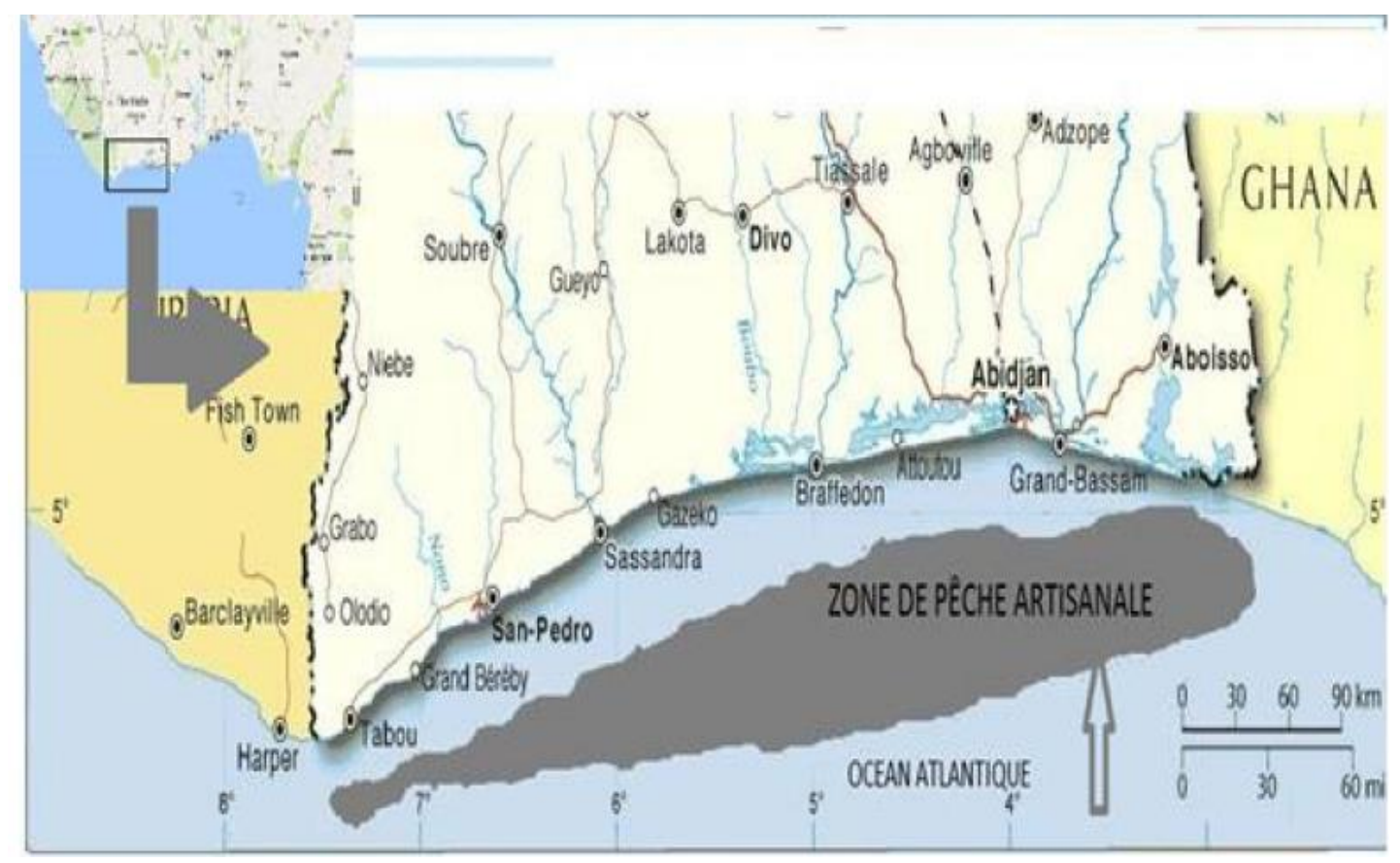

Figure 1 : Zone de pêche artisanale de Côte d'Ivoire.

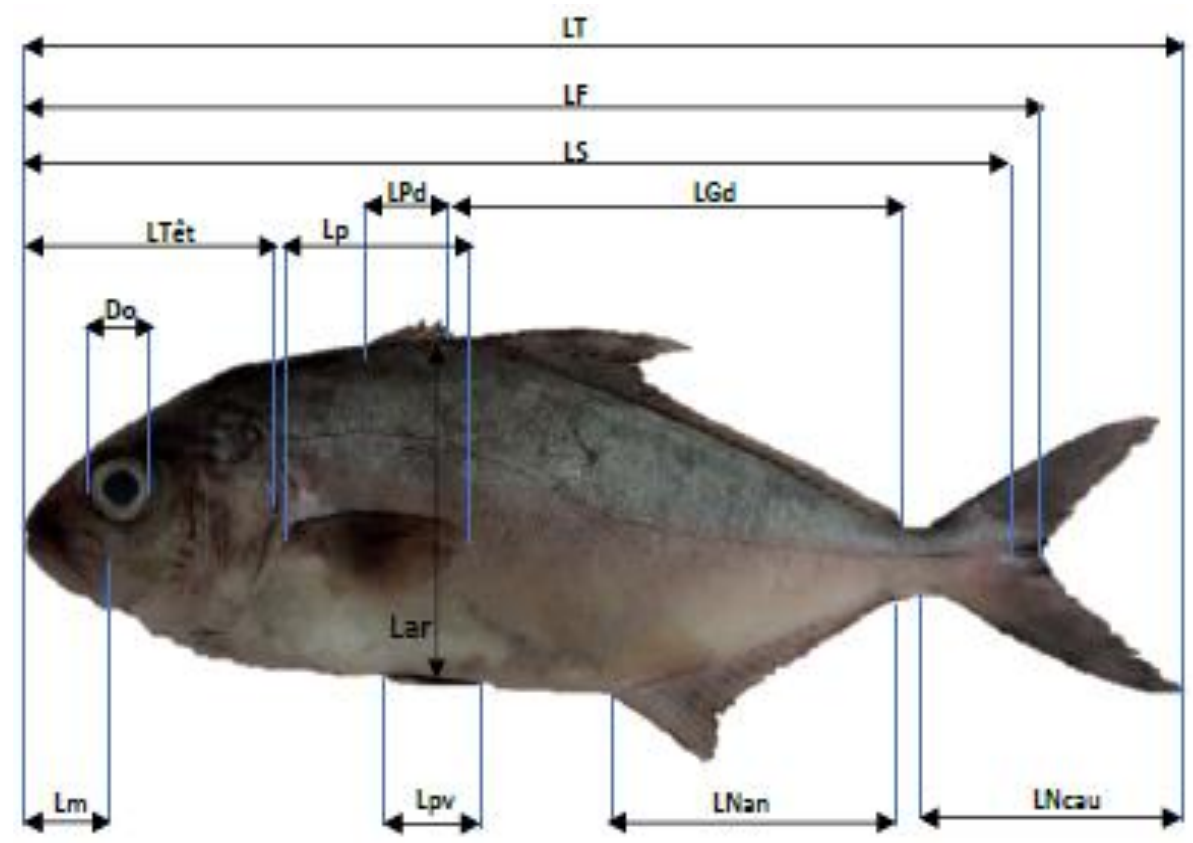

Figure 2 : mesures des paramètres morphologiques de Seriola carpenteri.

LT : longueur totale ; LF : longueur à la fourche ; LS : longueur standard ; LPd : longueur de la $1^{\text {ere }}$ nageoire dorsale ; LGd : longueur de la $2^{\text {ieme }}$ nageoire dorsale ; Lpv : longueur de la nageoire pelvienne ; Lp : longueur de la nageoire pectorale ; LNan : longueur de la nageoire anale ; LNcau : longueur de la nageoire caudale ; LTêt : longueur de la tête ; Lm : longueur du museau ; Do : diamètre de l'œil. 


\section{RESULTATS}

Caractérisation morphométrique de Seriola carpenteri échantillonné dans la Zone Economique exclusive de la côte d'ivoire d'août 2017 à juillet 2018

Le Tableau 1 présente les mesures des paramètres linéaires et pondéraux de la sériole guinéenne en fonction du sexe. Sur les 360 individus échantillonnés, 197 sont identifiés mâles contre 163 femelles. La comparaison des valeurs moyennes de ces différents paramètres, à l'aide du test $t$ de Student entre les deux sexes montre que seul le poids total enregistre une différence significative $(\mathrm{p}<0,05)$.

Caractéristiques allométriques de Seriola carpenteri échantillonné dans la zone exclusive économique de la côte d'ivoire d'août 2017 à juillet 2018

Le Tableau 2 présente l'étude biométrique de la population totale (mâles et femelles) de Seriola carpenteri échantillonné dans la Zone Economique Exclusive. La grande majorité des relations liants les paramètres étudiés à la longueur standard, prise comme longueur de référence, présentent une allométrie minorante $\left(b_{\text {observé }}<b_{\text {théorique }}=1\right.$ et $\mathrm{p}$ $<0,05)$. Cependant, les relations liants les paramètres tels que la longueur totale (LT), la longueur à la fourche (LF), la largeur du corps (Lar), la longueur de la $1^{\text {ère }}$ nageoire dorsale (LPd) et la longueur de la nageoire caudale (LNcau) à la longueur de référence (LS) enregistrent une isométrie de croissance $\left(b_{\text {observé }}=b_{\text {théorique }}={ }_{1}\right.$ et $\left.p>0,05\right)$. Ces résultats montrent par ailleurs des valeurs de corrélation
$\left(\mathrm{R}^{2}\right)$ supérieur à 0,60 , témoignant de l'existence d'une forte corrélation entre les paramètres étudiés et la longueur de référence (LS).

Caractéristiques méristiques de Seriola carpenteri échantillonné dans la zone exclusive économique de la côte d'ivoire d'août 2017 à juillet 2018 ( $N=360)$

Le Tableau 3 fait état des données méristiques quant aux rayons des différentes nageoires de Seriola carpenteri en fonction du sexe. Selon le tableau, aucune différence significative n'existe entre les données méristiques des nageoires des femelles et celles des mâles de Seriola carpenteri. En effet, le nombre moyen de rayons chez les mâles et les femelles pour la même nageoire est identique. Les valeurs moyennes enregistrées de la $1^{\text {ère }}$ nageoire dorsale, la $2^{\text {ème }}$ nageoire dorsale, la nageoire pectorale, la nageoire pelvienne et la nageoire anale sont identiques respectivement de $7 \pm 0,00 ; 31 \pm 0,87 ; 19 \pm 0,45 ; 12 \pm 0,80$ et $21 \pm 0,75 \mathrm{chez}$ les mâles et les femelles. Le nombre de rayons de la $2^{\text {ième }}$ nageoire dorsale se situe entre 29 et 34 et entre 29 et 31 respectivement chez le mâle et chez la femelle. Le nombre de rayons de la nageoire pectorale est compris entre 18 et 20 chez le mâle et entre 18 et $21 \mathrm{chez}$ la femelle. Le nombre de rayons de la nageoire anale et de la nageoire pelvienne sont compris respectivement entre 19 et 22 et entre 12 et 24 tant chez le mâle que chez la femelle. aucune différence significative n'a été observée entre le nombre de rayons des différentes nageoires chez le mâle et chez la femelle $(\mathrm{p}>0,05)$.

Tableau 1: Paramètres morphométriques de Seriola carpenteri échantillonné dans la Zone Economique Exclusive de la Côte d'Ivoire d'août 2017 à juillet 2018.

\begin{tabular}{lcccccc}
\hline \multicolumn{1}{c}{ Paramètres } & Sexe & Nombre & Minimum & $\begin{array}{c}\text { Maximu } \\
\mathbf{m}\end{array}$ & $\begin{array}{c}\text { Moyenne } \pm \\
\text { Ecart-type }\end{array}$ & Significativité \\
\hline Longueur totale & M & 197 & 30,7 & 42,3 & $35,6 \pm 2,5$ & NS \\
Longueur & F & 163 & 29,7 & 44,3 & $35,9 \pm 2,8$ & \\
standard & M & 197 & 22,8 & 35 & $29,5 \pm 2,1$ & NS \\
Longueur à la & F & 163 & 24,8 & 36,2 & $29,8 \pm 2,2$ & \\
fourche & M & 197 & 26,4 & 36,2 & $30,9 \pm 2,2$ & NS \\
Poids total & F & 163 & 25,6 & 38,3 & $31,2 \pm 2,4$ & S \\
& M & 197 & 300,2 & 935 & $516,4 \pm 116,0$ &
\end{tabular}




\begin{tabular}{|c|c|c|c|c|c|c|}
\hline \multirow{3}{*}{ Largeur } & $\mathrm{F}$ & 163 & 310,2 & 919,5 & $523,8 \pm 128,7$ & \multirow{3}{*}{ NS } \\
\hline & M & 197 & 9,4 & 14,5 & $11,1 \pm 0,9$ & \\
\hline & $\mathrm{F}$ & 163 & 9,4 & 14,7 & $11,3 \pm 1,1$ & \\
\hline \multirow{2}{*}{$\begin{array}{l}\text { Longueur de la } \\
\text { tête }\end{array}$} & M & 197 & 7,4 & 10,6 & $8,5 \pm 0,5$ & \multirow{2}{*}{ NS } \\
\hline & $\mathrm{F}$ & 163 & 7,9 & 10 & $8,6 \pm 0,4$ & \\
\hline \multirow{2}{*}{$\begin{array}{l}\text { Longueur de la } 1^{\text {ere }} \\
\text { nageoire dorsale }\end{array}$} & M & 197 & 2,4 & 3,6 & $2,9 \pm 0,2$ & \multirow{2}{*}{ NS } \\
\hline & $\mathrm{F}$ & 163 & 2,4 & 3,7 & $3,0 \pm 0,2$ & \\
\hline \multirow{2}{*}{$\begin{array}{l}\text { Longueur de la } \\
2^{\text {eme }} \text { nageoire } \\
\text { dorsale }\end{array}$} & M & 197 & 12,8 & 14,1 & $13,4 \pm 0,2$ & \multirow[b]{2}{*}{ NS } \\
\hline & $\mathrm{F}$ & 163 & 10,9 & 16,3 & $13,2 \pm 1,0$ & \\
\hline \multirow{2}{*}{$\begin{array}{l}\text { Longueur de la } \\
\text { nageoire pectorale }\end{array}$} & M & 197 & 4,4 & 5,1 & $4,7 \pm 0,1$ & \multirow{2}{*}{ NS } \\
\hline & $\mathrm{F}$ & 163 & 4,1 & 5,7 & $4,8 \pm 0,3$ & \\
\hline \multirow{2}{*}{$\begin{array}{l}\text { Longueur de la } \\
\text { nageoire pelvienne }\end{array}$} & M & 197 & 5,4 & 6,1 & $5,7 \pm 0,1$ & \multirow{2}{*}{ NS } \\
\hline & $\mathrm{F}$ & 163 & 5,1 & 6,7 & $5,8 \pm 0,3$ & \\
\hline \multirow{2}{*}{$\begin{array}{l}\text { Longueur de la } \\
\text { nageoire anale }\end{array}$} & M & 197 & 6,9 & 9,7 & $8,2 \pm 0,5$ & \multirow{2}{*}{ NS } \\
\hline & $\mathrm{F}$ & 163 & 6,9 & 9,7 & $8,1 \pm 0,5$ & \\
\hline \multirow{2}{*}{$\begin{array}{l}\text { Longueur de la } \\
\text { nageoire caudale }\end{array}$} & M & 197 & 6,6 & 8,1 & $7,3 \pm 0,3$ & \multirow{2}{*}{ NS } \\
\hline & $\mathrm{F}$ & 163 & 5,3 & 9,5 & $7,1 \pm 0,8$ & \\
\hline \multirow{2}{*}{$\begin{array}{l}\text { Longueur du } \\
\text { museau }\end{array}$} & M & 197 & 3,1 & 3,4 & $3,2 \pm 0,1$ & \multirow{2}{*}{ NS } \\
\hline & $\mathrm{F}$ & 163 & 2,6 & 4 & $3,2 \pm 0,2$ & \\
\hline \multirow{2}{*}{ Diamètre de l'œil } & M & 197 & 1,7 & 1,9 & $1,8 \pm 0,1$ & \multirow{2}{*}{ NS } \\
\hline & $\mathrm{F}$ & 163 & 1,6 & 1,9 & $1,7 \pm 0,1$ & \\
\hline
\end{tabular}

(S) : significatif et (NS) : non significatif

Tableau 2 : Nature de l'allométrie et de la corrélation entre les paramètres morphométriques et la longueur standard (LS) chez Seriola carpenteri échantillonné dans la Zone Economique Exclusive de la Côte d'Ivoire d'août 2017 à juillet $2018(\mathrm{~N}=360)$.

\begin{tabular}{lccccc}
\hline Relations morphométriques & Equation & $\mathbf{R}^{\mathbf{2}}$ & t-test & $\mathbf{P}>\mathbf{t}$ & Type d'allométrie \\
\hline PT/LS & $\mathrm{y}=0,04 \mathrm{x}^{2,80}$ & 0,81 & 126,50 & $\mathrm{~S}$ & Minorante \\
LT/LS & $\mathrm{y}=1,28 \mathrm{x}^{0,98}$ & 0,93 & 54,60 & $\mathrm{NS}$ & Isométrie \\
LF/LS & $\mathrm{y}=1,08 \mathrm{x}^{0,99}$ & 0,95 & 32,77 & $\mathrm{NS}$ & Isométrie \\
Lar/LS & $\mathrm{y}=0,29 \mathrm{x}^{1,08}$ & 0,81 & $-130,96$ & $\mathrm{NS}$ & Isométrie \\
LTêt/LS & $\mathrm{y}=0,73 \mathrm{x}^{0,73}$ & 0,79 & 623,78 & $\mathrm{~S}$ & Minorante \\
LPd/LS & $\mathrm{y}=0,07 \mathrm{x}^{1,09}$ & 0,93 & $-249,72$ & $\mathrm{NS}$ & Isométrie \\
LGd/LS & $\mathrm{y}=1,64 \mathrm{x}^{0,62}$ & 0,64 & 694,66 & $\mathrm{~S}$ & Minorante \\
Lpect/LS & $\mathrm{y}=0,58 \mathrm{x}^{0,62}$ & 0,84 & 1200,68 & $\mathrm{~S}$ & Minorante \\
Lpev/LS & $\mathrm{y}=1,11 \mathrm{x}^{0,49}$ & 0,79 & 1780,50 & $\mathrm{~S}$ & Minorante \\
LNan/LS & $\mathrm{y}=0,39 \mathrm{x}^{0,89}$ & 0,90 & 306,29 & $\mathrm{~S}$ & Minorante \\
LNcau/LS & $\mathrm{y}=0,25 \mathrm{x}^{0,99}$ & 0,72 & 16,98 & $\mathrm{NS}$ & Isométrie \\
Do/LS & $\mathrm{y}=0,85 \mathrm{x}^{0,21}$ & 0,1457 & 1342,82 & $\mathrm{NS}$ & Minorante \\
Lm/LS & $\mathrm{y}=0,36 \mathrm{x}^{0,65}$ & 0,63 & 603,90 & $\mathrm{~S}$ & Minorante \\
\hline
\end{tabular}

(S) : significatif et (NS) : non significatif 
Tableau 3 : Données méristiques des rayons des nageoires de $S$. carpenteri échantillonné dans la Zone Economique Exclusive de la Côte d'Ivoire d'août 2017 à juillet 2018.

\begin{tabular}{lcccccc}
\hline \multicolumn{1}{c}{ Paramètres } & Sexes & $\begin{array}{c}\text { Effectif } \\
\text { par sexe }\end{array}$ & Minimum & Maximum & Moy \pm ET & $p$-value \\
\hline Nombre de rayons de & M & 197 & 7 & 7 & $7,00 \pm 0,00$ & $P>0,05$ \\
la 1 ${ }^{\text {ere }}$ nageoire dorsale & F & 163 & 7 & 7 & $7,00 \pm 0,00$ & \\
Nombre de rayons la & M & 197 & 29 & 34 & $31 \pm 1,12$ & $P>0,05$ \\
$2^{\text {ieme }}$ nageoire dorsale & F & 163 & 29 & 31 & $31 \pm 0,61$ & \\
Nombre de rayons la & M & 197 & 18 & 20 & $19 \pm 0,38$ & $P>0,05$ \\
nageoire pectorale & F & 163 & 18 & 21 & $19 \pm 0,51$ & \\
Nombre de rayons la & M & 197 & 12 & 14 & $12 \pm 0,82$ & $P>0,05$ \\
nageoire pelvienne & F & 163 & 12 & 14 & $12 \pm 0,78$ & \\
Nombre de rayons la & M & 197 & 19 & 22 & $21 \pm 0,81$ & $P>0,05$ \\
nageoire anale & F & 163 & 19 & 22 & $21 \pm 0,69$ & \\
\hline
\end{tabular}

\section{DISCUSSION}

La caractérisation biométrique de la sériole guinéenne (Seriola carpenteri) échantillonnée dans la Zone Economique Exclusive de la Côte d'Ivoire a nécessité une analyse morphométrique et méristique de 360 spécimens de la sériole guinéenne, Seriola carpenteri dont 197 mâles et 163 femelles. Il ressort qu'aucun dimorphisme sexuel ne peut être établi à partir des paramètres mesurés qui sont statistiquement identiques chez les deux sexes. Ainsi, les relations morphométriques chez la population totale de la sériole guinéenne montrent d'une part que la vitesse de croissance de la longueur standard est plus rapide que la vitesse de la croissance de la longueur du museau, la longueur de la tête, la longueur des nageoires (grande nageoire dorsale, pectorale, pelvienne et anale), le diamètre de l'œil et le poids et d'autres part, que la longueur totale, la longueur à la fourche, les longueurs des nageoires (petite dorsale et caudale) ont une vitesse de croissance proportionnellement égale à celle de la longueur standard. Les spécimens de $S$. carpenteri présentent une croissance allométrique minorante $(\mathrm{b}=2,80<3)$. Cela implique une augmentation de la longueur par rapport au poids au fur et à mesure de la croissance de l'espèce. Nos résultats concordent avec ceux de Magnusson et Magnusson (1987), Manooch et Potts (1997) et Kozul et al. (2001). Ces auteurs ont obtenu des résultats similaires chez $S$. dumerili une espèce du même genre et ont enregistré respectivement 2,83 dans les îles de Cap-Vert, 2,86 dans le golfe du Mexique et 2,88 dans le sud-est de l'Adriatique. Le nombre des différentes nageoires comme la $1^{\text {ere }}$ et la $2^{\text {eme }}$ nageoire dorsale, la nageoire pectorale, la nageoire ventrale, la nageoire anale et la nageoire caudale ne sont pas significativement différents chez les mâles et les femelles. Le nombre de rayons de la $1^{\text {ère }}$ nageoire dorsale est de 7 chez le mâle et chez la femelle. Ces résultats corroborent ceux de Pizzicori et al. (2000), Quéro (1986) et Louisy (2015) ; qui ont fait des travaux similaires sur la même espèce et ont observés respectivement; 7 à Lampedusa Island dans la Méditerranée, 7 dans l'Atlantique Nord-Est, et 7 à 8 en Méditerranée. Par ailleurs le nombre de rayons est inférieure à celui obtenu par Capapé et al. (2018) au Nord-Est de la Tunisie et sur les côtes sénégalaises chez la même espèce. Le nombre de rayons de la $2^{\text {ième }}$ nageoire dorsale 
est compris entre 28 et 34 chez le mâle et entre 28 et 31 chez la femelle. Ces résultats sont en accord avec ceux de Capapé et al. (2018) et Smith-Vaniz (1986). Ces auteurs ont respectivement observé chez la même espèce 30 à 33 et 28-33 rayons. Le nombre de rayons de la nageoire pectorale est compris entre 18 et 20 chez le mâle et entre 18 et 21 chez la femelle. Ces résultats confirment ceux de Pizzicori et al. (2000) et Capapé et al. (2018) qui ont obtenus le même résultat à savoir 20 rayons à la nageoire pectorale chez la même espèce. Le nombre de rayons de la nageoire anale est compris entre 19 et 22 chez le mâle et chez la femelle. Nos résultats sont conformes avec ceux obtenus par Pizzicori et al. (2000), Quéro (1986) et Capapé et al. (2018). En effet, ces auteurs ont respectivement obtenu 19, 21 et 21 rayons chez la même espèce.

Le nombre de rayons de la nageoire pelvienne est compris entre 6 et 12 chez le mâle et chez la femelle. Ces résultats confirment ceux de Capapé et al. (2018) qui ont obtenu chez la même espèce 6 rayons à la nageoire pelvienne.

\section{Conclusion}

Cette étude a montré qu'aucun dimorphisme sexuel n'existe entre les mâles et les femelles de la sériole guinéenne de la Zone Economique Exclusive de la Côte d'Ivoire. Cependant dans cette zone, l'espèce est caractérisée par une allométrie minorante des relations taille-poids et une isométrie des relations entre les paramètres linéaires majeurs. Au terme de cette étude, il serait intéressant de mener des études complémentaires visant à caractériser le cycle sexuel chez cette espèce afin de connaitre sa stratégie de reproduction.

\section{CONFLIT D'INTÉRÊTS}

Tous les auteurs de ce manuscrit sont unanimes, aucun conflit d'intérêts ne s'oppose à la publication de cet article.

\section{CONTRIBUTIONS DES AUTEURS}

LYAA est l'investigateur principal ; YLA et JNY ont contribué à la récolte des spécimens et coordonné tous les travaux au laboratoire d'Anatomie Pathologique du CHU de Treichville. MCB et KY ont proposé le protocole de recherche et sont les co-directeurs du travail.

\section{REMERCIEMENTS}

Nous remercions les auteurs de cette étude, le Laboratoire de Biologie et Cytologie animales, le laboratoire d'Anatomie pathologie (AnaPath) du Centre Hospitalier Universitaire de Treichville et au Centre de Recherches Océanologiques.

\section{REFERENCES}

Bijaksana U, Hidayaturrahmah, Sari DDK. 2015. Re-stocking' model of snakehead farming, Channa striata blkr in the swamp bangkau of South Kalimantan Province. Global Journal of Fisheries and Aquaculture, 3(2): 198-204.

Capapé C, Rafrafi-Nouira S, Diatta Y, Golani D. 2018. On the Mediterranean occurrence of Guinean amberjack Seriola carpenteri (Osteichthyes: Carangidae), with first confirmed record from the Tunisian coast. Cahier de Biologie Marine, 59 : 399-402. DOI: 10.21411/CBM.A.FAC33F0D

Diaha CN, N'da K, Soro Y, 2010. Régime alimentaire de Scomberomorus tritor (Cuvier, 1831) dans le Golfe de Guinée. International Journal of Biological and Chemical Sciences, 4(3): 669-680. DOI: 10.4314/ijbcs.v4i3.60482

Golani D, Orsi-Relini L, Massuti E, Quignard J-P. 2002. In CIESM atlas of exotic species in the Mediterranean: Fishes. Ed. by BRIAND F. CIESM Publishers, Monaco.

FIRCA. 2013. La filière du progrès, ACTE 11; à la découverte de la filière pêche et aquaculture, $44 \mathrm{p}$.

Kozul V, Skaramuca B, Kraljevi M, Dul JI, Glamuzina B. 2001. Age, croissance et mortalité de l'amberjack mediterranéen, Seriola dumerili (Risso., 1810), originaire $\mathrm{du}$ sud-est de l'Adriatique. Journal of Applied Ichthyology, 17: 134-141.

Le Cren ED. 1951. The length-weight relationship and seasonal cycle in gonad 
weight and condition in the perch (Perca fluviatilis). Journal of Animal Ecology, 20: 201-219. DOI: http: //dx.doi.org/10.2307/1540

Louisy P. 2015. Guide d'identification des poissons de mer d'Europe et de la Méditerranée, 512p.

Magnusson J, Magnusson JVV. 1987. ICEIDA / Projet de pêche dans les îles du Cap-Vert. Enquête sur les ressources halieutiques démersales dans les eaux aux large des îles du Cap-Vert. IV Rapport : résumé des informations sur les espèces. Agences islandaises de développement international / Institut de recherche marine. $114 \mathrm{p}$.

Manooch CS III, Potts JC. 1997. Age, croissance et mortalité du plus grand amberjack, Seriola dumerili, issu de la pêcherie de headboat du golfe de Mexique. Taureau. Bulletin of Marine Science, 61(3) : 671 - 683.

Mylonas CC, Fostier A, Zanny S. 2010. Broodstock management and hormonal manipulations of fish reproduction. General and Comparative Endocrinology, 165(3): 516-534. DOI: 10.1016/j.ygcen.2009.03.007.

Niass F, Fall S. 2015. Reproduction and larval rearing of the fresh water prawn, Macrobrachium vollenhovenii in Sénégal. International Journal of Biological and Chemical Sciences, 9(6): 2523-2534. DOI: 10.4314/ijbcs.v9i6.2

Pizzicori P, Castriota L, Marino G, Andaloro F. 2000. Seriola carpenteri a new immigrant in the Mediterranean from the Atlantic Ocean. Journal of Fish Biology, 57: 13351338. DOI: $10.1006 /$ jfbi.2000.1392
Kamelan TM, Berté S, N'Zi GK, Bamba M, Bi GG, Kouamélan EP. 2013. Peuplement ichtyologique du complexe Brimé-MénéNounoua, Côte d'Ivoire (Afrique de l'Ouest). International Journal of Biological and Chemical Sciences, 7(6): 2248-2263. DOI: 10.4314/ijbcs.v7i6.6

Karatas M. 2005. Research Techniques in Fish Biology. Nobel Press: Ankara ; Turkey; 498p.

N'goran YN, Amon KJB, Bard FX. 2001. Captures d'istiophoridés (voiliers Istiophorus albicans, marlin bleu Makaira nigricans, marlin blanc Tetrapturus albidus) et effort de pêche des filets maillants dérivants en Côte d'Ivoire SCRS/00/63. Collective Volume of Scientific Papers., ICCAT, 53: 272-280.

Ouedraogo R, Soara AE, Zerbo H. 2015. Caractérisation du peuplement piscicole du réservoir de Boalin, Ziniaré (Burkina Faso) deux décennies après l'introduction de Hétérotis niloticus. International Journal of Biological and Chemical Sciences, 9(5): 2488-2499. DOI: 10.4314/ijbcs.v9i5.20

Quéro, JC. 1986. Capture dans le golfe de Gascogne de Seriola carpenteri Mather, 1971 (Pisces, Perciformes, Carangidae), espèce nouvelle pour la faune de l'Atlantique nord-est. Cybium, 10 : 302304.

Smith-Vaniz WF. 1986. Carangidae. In Fishes of the North-western Atlantic and the Mediterranean (Vol. II), Whitehead PJP, Bauchot ML, Hureau JC, Nielsen JJ, Tortonese E (eds). UNESCO: Paris; 815844. 